Trauma Berufskrankh 2007 - 9 [Suppl 2]:S202-S209 DOI 10.1007/s10039-006-1163-6

Online publiziert: 19. August 2006

๑) Springer Medizin Verlag 2006

S. Fuchs ${ }^{1} \cdot$ S. Wallstabe ${ }^{1} \cdot$ U. Gerlach ${ }^{1} \cdot$ M. E. Wenzl ${ }^{2} \cdot$ C. Jürgens ${ }^{1,2}$

${ }^{1}$ Abteilung für Unfall- und Wiederherstellungschirurgie,

BG-Unfallkrankenhaus, Hamburg

${ }^{2}$ Abteilung für Unfall- und Wiederherstellungschirurgie,

Universitätsklinikum Schleswig-Holstein, Campus Lübeck, Lübeck

\title{
Sekundäreingriffe nach Ellengelenkverletzungen
}

Im Folgenden soll auf die überwie-

Aufgrund der Komplexität des Ellenbogengelenks und dessen Verletzungen ist insbesondere nach operativen Versorgungen mit Komplikationen zu rechnen. Diese führen häufig zu funktionellen Einbußen mit Bewegungseinschränkung des Gelenks. Somit sind Sekundäreingriffe vorprogrammiert.

Ursächlich für posttraumatische Bewegungseinschränkungen des Ellenbogengelenks sind im Wesentlichen

- die Kapselfibrose mit z. T. kombinierten heterotrophen Ossifikationen,

- knöcherne Fehlheilungen in Form von Pseudarthrosen oder Achsfehlstellungen,

- Gelenkdefekte und

- posttraumatische Arthrosen.

Des Weiteren führen Infektionen des Ellenbogengelenks bei zu später Diagnostik und Therapie zu hochgradigen Funktionsverlusten mit z. T. erheblichen Gelenkdestruktionen.

Im eigenen Patientengut der letzten 10 Jahre fanden sich insgesamt 790 sekundäre Operationen bei 516 Patienten nach primär operativer Intervention am Ellenbogengelenk. Den größte Anteil davon machten Materialentfernungen aus. Es wurden aber auch 165 Arthrolysen, in der Regel in Kombination mit einer Neurolyse des N. ulnaris, durchgeführt. Des Weiteren ergaben sich 111 Pseudarthrosen- bzw. Fehlstellungsbehandlungen bei 33 Patienten. Weiterhin wurde bei 42 Patienten eine Behandlung bei Ellengelenkempyem oder Osteitis erforderlich (- Tab. 1). gend durchgeführten Operationen eingegangen werden.

\section{Arthrolyse}

Die exakte Inzidenz posttraumatischer Funktionsstörungen nach Verletzungen des Ellenbogengelenks ist unbekannt. Nach Sojbjerg [26] wird davon ausgegangen, dass 5\% aller knöchernen Ellenbogenverletzungen zu einer posttraumatischen Gelenksteife führen.

\section{Ursachen und Häufigkeit}

Hinsichtlich der Ätiologie von Ellengelenkeinsteifungen wird üblicherweise in extrinsische/extraartikuläre und intrinsische/intraartikuläre Ursachen eingeteilt. $\mathrm{Zu}$ Letzteren werden posttraumatische Gelenkstufenbildungen, Osteophyten, freie Gelenkkörper, eine Fibrosierung der Gelenkkapsel und sekundäre degenerative Veränderungen der Gelenkfläche gezählt. Extraartikuläre Veränderungen imponieren als Vernarbungen in der Muskulatur, Adhäsionen in den Gleitschichten der Muskeln, Sehnen und Bänder, Narbeneinziehungen nach Operationen und Verbrennungen sowie extrakapsuläre Fibrosierungen und heterotrope Ossifikationen (\ Abb. 1).

Die Inzidenz von Ellenbogensteifen nach Schädigung unterschiedlichen Ausmaßes ist bis heute nicht eindeutig geklärt. Mohan [18] konnte in der Aufarbeitung von 200 Fällen posttraumatischer Ellenbogensteifen zeigen, dass di- ese in 38\% mit Luxationsfrakturen des Gelenks, in $20 \%$ mit reinen Luxationen und in $10 \%$ mit Radiusköpfchenfrakturen zusammenhingen. Die knöchernen Verletzungen sowie die begleitenden Bandverletzungen sind jedoch nur ein Aspekt in der Entwicklung der posttraumatischen Ellenbogensteife. Lang dauernde Immobilisation des Gelenks mit Verklebung der Gleitschichten, Verkürzungen von Kapsel- und Bandstrukturen sowie intraartikuläre Adhäsionen tragen in der Folge zur Bewegungseinschränkung bei. Laut Loew [13] sollte die Dauer der Ruhigstellung des Ellenbogengelenks nicht über 3 Wochen betragen.

\section{Klassifikationen der Ellengelenksteife}

Sie orientieren sich an den Bewegungsmöglichkeiten. Sie haben meist nur deskriptiven Charakter und sind für die Therapieentscheidung von untergeordneter Bedeutung. Die Klassifikation nach Hastings u. Graham [4] differenziert Ein-

\begin{tabular}{|lc|}
\hline $\begin{array}{l}\text { Tab. } 1 \\
\text { lenk von 1995-2005 }\end{array}$ \\
\hline Materialentfernung & 334 \\
\hline Arthrolyse/Neurolyse & 165 \\
\hline Pseudarthrose & 111 \\
\hline Fehlstellung & 33 \\
\hline Empyembehandlung & 42 \\
\hline Arthroskopie & 31 \\
\hline Narkosemobilisation & 18 \\
\hline Sonstige & 56 \\
790 Sekundäreingriffe bei 516 Patienten \\
\hline
\end{tabular}




\begin{tabular}{|c|c|}
\hline Grad & Ausmaß \\
\hline I & Kein Bewegungslimit \\
\hline Ila & $\begin{array}{l}\text { Subtotale Limitierung Flexion/Ex- } \\
\text { tension }\end{array}$ \\
\hline $\mathrm{Ilb}$ & $\begin{array}{l}\text { Subtotale Limitierung Pronation/Su- } \\
\text { pination }\end{array}$ \\
\hline Ilc & Kombination \\
\hline III & Ankylose mit Brückenbildung \\
\hline
\end{tabular}

Tab. 3 Klassifikation der Ellengelenksteife nach Jäger $u$. Wirth [6]

\begin{tabular}{|ll}
\hline Klassifikation & $\begin{array}{l}\text { Ausmaß (Umfang } \\
\text { der Gesamtbeweg- } \\
\text { lichkeit) }\end{array}$ \\
\hline Gering & $>90^{\circ}$ \\
Mäßig & $60-90^{\circ}$ \\
Schwer & $30-60^{\circ}$ \\
\hline Sehr schwer & $<30^{\circ}$ \\
\hline
\end{tabular}

schränkungen in der Flexions-/Extensionsebene sowie in der Pro- und Supinationsebene (- Tab. 2). Die Einteilung nach Jäger u. Wirth [6] unterscheidet den Umfang der Gesamtbeweglichkeit bei Beugung und Streckung (• Tab.3).

\section{Behandlungsstrategien}

Sie richten sich nach dem Umfang der Beweglichkeit. Es muss die Frage gestellt werden, wie viel Beweglichkeit das Individuum im Alltag benötigt. Morrey [19] und Morrey et al. [22] beschrieben, dass der Mensch eine Beweglichkeit von $\mathrm{o} / 30 / 130^{\circ}$ für Streckung und Beugung sowie $50 / 0 / 50^{\circ}$ für Pro- und Supination bei $90 \%$ seiner täglichen Aktivitäten benötigt („funktionell arc" bzw. funktioneller Bogen nach Morrey). Einschränkungen der Beweglichkeit in Bezug auf die Extension werden eher toleriert als ein gleich großer Ausfall der Flexion.

Exner et al. [3] schlugen vor, ein Bewegungsausmaß von $\mathrm{o} / 30 / 110^{\circ}$ als guten Standard anzustreben. Dieses gilt als Orientierung, bedarf aber einer individuellen Betrachtung, insbesondere beim bilateralen Befall sowie z. B. beim Musiker. Der Geigenspieler muss seinen Arm vollständig strecken können.

Trauma Berufskrankh 2007 · 9 [Suppl 2]:S202-S209 DOI 10.1007/s10039-006-1163-6

(c) Springer Medizin Verlag 2006

\section{S. Fuchs · S. Wallstabe $\cdot$ U. Gerlach $\cdot$ M. E. Wenzl $\cdot$ C. Jürgens Sekundäreingriffe nach Ellengelenkverletzungen}

\section{Zusammenfassung}

Aus operativen Versorgungen von Ellenbogengelenkverletzungen resultierende Komplikationen führen zu funktionellen Einbußen mit Einschränkungen im privaten und beruflichen Leben. Die offene Arthrolyse in Kombination mit Neurolyse des N. ulnaris sollte frühzeitig bei knöcherner Stabilität mit einem angestrebten Bewegungsausmaß von $0 / 30 / 110^{\circ}$ für Streckung und Beugung erfolgen. Eine arthroskopische Arthrolyse ist nur ausnahmsweise bei milden Bewegungseinschränkungen sowie freien Gelenkkörpern sinnvoll. Die Pseudarthrosenbehandlung oder Korrektur von Achsfehlstellungen am Ellenbogengelenk stellen eine wichtige Operationsindikation dar. Ellengelenkempy- eme nach Trauma und operativer Behandlung müssen frühzeitig und konsequent behandelt werden. Die Therapie erfolgt stadienadaptiert und in der Regel offen, wobei immer ein Funktionserhalt angestrebt werden sollte. Die Indikation zum endoprothetischen Ersatz des posttraumatisch veränderten Ellenbogengelenks sollte streng gestellt werden. Sekundäreingriffe am Ellenbogengelenk erfordern eine individuelle Behandlungsstrategie und ein auf den einzelnen Patienten abgestimmtes Operationsverfahren.

\section{Schlüsselwörter}

Ellenbogengelenk · Sekundäreingriffe · Offene Arthrolyse · Pseudarthrosen · Empyem

\section{Secondary interventions after injuries to the elbow}

\section{Abstract}

complications of surgical treatment of elbow joint injuries can lead to functional defects affecting both private and professional life. Open arthrolysis together with neurolysis of the ulnar nerve should be performed at an early stage providing the bone is stable, And every effort should be made to achieve a range of movement encompassing $0-30$ 110 degrees for extension and flexion $\mathrm{Ar}-$ throscopic arthrolysis only makes sense in exceptional cases of mildly restricted range of movement or loose joint bodies. Treatment of pseudarthrosis and correction of displacements are important indications for surgery. Elbow empyema following trauma and operative intervention require systematic treat- ment as early as possible. The treatment is tailored to the stage of the infection and usually takes the form of open surgery; efforts should always be made to preserve joint function. Endoprothetic substitution of posttraumatically altered elbow joints should be subject to strict observation of the indications. Secondary interventions at the elbow require individual treatment strategies and surgery tailored to the individual patient's particular situation.

\section{Keywords}

Elbow joint · Secondary interventions · Open arthrolysis · Pseudarthrosis · Empyema 


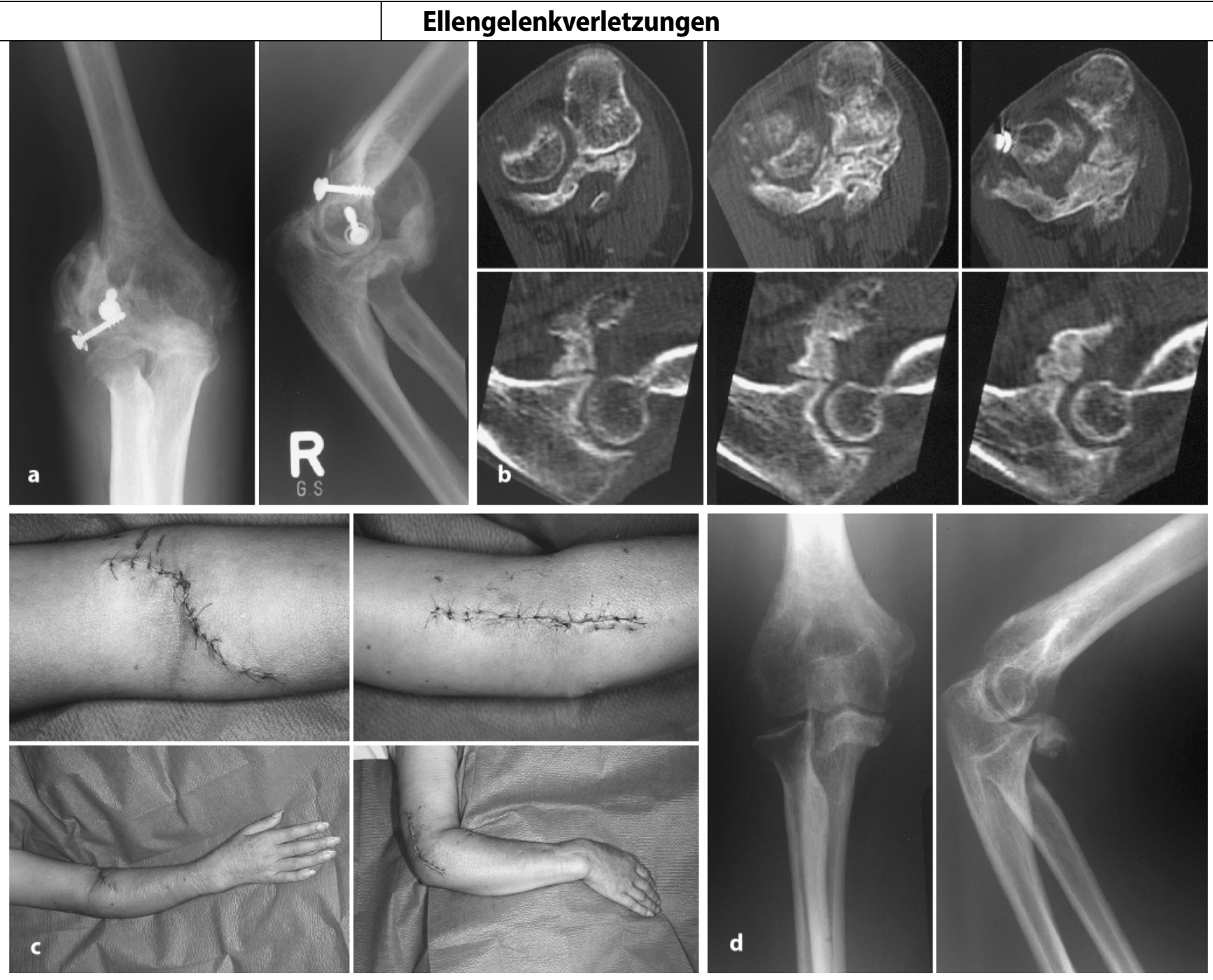

Abb. 1 \ 50-jährige Patientin, a Ankylose des Ellenbogengelenks nach Luxation und Abrissfraktur des radialen Epikondylus, b CT, ausgeprägte ventrale Ossifikationen, $\mathbf{c}$ intraoperativer Situs mit lateralem und ventralem Zugang, d radiologischer Befund postoperativ bei einer Beweglichkeit von 0/10/115

\section{Operation}

Vor der Indikationsstellung zur operativen Arthrolyse sollten die konservativen physiotherapeutischen Anwendungen ausgereizt worden sein $[1,8]$. Kommt es trotz regelmäßiger und vollständig durchgeführter Physiotherapie zu keinem Bewegungsgewinn oder sogar $\mathrm{zu}$ einer Abnahme der Beweglichkeit, sollte die operative Intervention in Erwägung gezogen werden. Der Zeitpunkt ist mit 4-6 Monaten nach dem Unfallereignis oder der Erstversorgung bei knöcherner Stabilität gegeben. Je länger man wartet und insbesondere die 9-Monats-Grenze überschreitet, desto schlechter werden die Ergebnisse. Das Abwarten der Ausreifung der Ossifikationen oder aber eine völlig unauffällige alkalische Phosphata- se sollten nicht abgewartet werden, da damit häufig der zeitliche Rahmen überzogen wird [14].

Als operative Verfahren der Arthrolyse am Ellenbogengelenk kommen zum einen die Arthroskopie, die offene Arthrolyse oder aber auch die Distraktionsarthrolyse in Frage.

Arthroskopische Verfahren. Die Arthroskopie des Ellenbogengelenks als Methode zur Arthrolyse stellt eher eine untergeordnete Rolle dar, da wesentliche Bereiche des Gelenks nicht oder nur schwer einsehbar sind. Insbesondere bei Arthrofibrose reduziert sich das intraartikuläre Volumen, sodass der Bewegungsspielraum für das Arthroskop und resezierende Instrumente deutlich eingeschränkt ist. Die aufwändige Darstellung benötigt Zeit und bedingt durch den Wasseraustritt eine zunehmende Weichteilschwellung. Aus diesen Gründen sehen wir die Indikation zur Arthroskopie nur bei milden Kontrakturen, bei Narbensträngen, zur Bergung freier Gelenkkörper und bei der reaktiven Synovitis. Des Weiteren können lokale Osteophyten im Bereich des Processus coronoideus oder auch des Olekranons abgetragen werden [30].

Offene Arthrolyse. Diese Technik wurde am Ellengelenk vielfach beschrieben [5, 10, 15, 17, 20]. Der Zugang orientiert sich an vorbestehenden Narben, der vorherrschenden Pathomorphologie sowie möglichen Veränderungen an den umgebenen Weichteilen und einer begleitenden neurologischen Symptomatik. 
Klassisch wird der Eingriff nach Morrey [20] über einen limitierten radialen Zugang mit Arthrotomie, Durchtrennung der ventralen und dorsalen Kapsel sowie Entfernung von Osteophyten durchgeführt („column procedure“) $[15,17]$. Nach Lahoda et al. [10] sollte zur Erzielung einer freien bzw. fast freien Beugung regelhaft eine zusätzliche Neurolyse des N. ulnaris über einen medialen Zugang erfolgen, um einem Impingement des Nervs vorzubeugen.

Aufgrund von Voroperationen nach Frakturversorgung des distalen Oberarms sowie des Olekranons findet sich häufig ein vorgegebener dorsaler Zugang, sodass die Arthrolyse auch von dorsal erfolgen kann. Dabei kann zunächst der N. ulnaris im Sinne einer Neurolyse präpariert werden. Anschließend werden der humeroradiale Gelenkabschnitt sowie der dorsale im Bereich der Fossa olecrani aufgesucht und entsprechende Osteophyten oder Adhäsionen entfernt.

Distraktionsarthrolyse. Die Methode stellt eine Alternative zur offenen Arthrolyse des Ellenbogen dar. Die intraoperative Gelenkdistraktion wird zur allmählichen Dehnung der geschrumpften Kapsel-Band-Strukturen eingesetzt und erlaubt eine sichere Separation der Gelenkflächen [14, 24]. Ihr folgt eine Relaxationsphase mit anschließender fixateurgesteuerter Mobilisationsphase. Hierzu stehen verschiedene Möglichkeiten wie Ringfixateur- oder Orthofixateursysteme zur Verfügung. Eigene Erfahrungen in der geschlossenen Arthrolyse im Bewegungsfixateur bestehen am Ellenbogen nicht. Vielmehr wird in unserer Klinik der Fixateur externe in Verbindung mit dem Hexapoden in der postoperativen Mobilisationsbehandlung eingesetzt $(\boldsymbol{\bullet}$ Abb. 3c, d).

\section{Pseudarthrosen}

Sie sind im Ellenbogenbereich aufgrund der guten Heilungstendenz im epi- und metaphysären Anteil selten [2, 25]. Nur in wenigen Fällen sind präoperative Komplikationen oder andere Begleitumstände dafür verantwortlich. Meist sind sie Folge einer unsachgemäßen Therapie in Form einer konservativen Behandlung oder einer instabilen Osteosynthese (• Abb. 2) [2, 25].
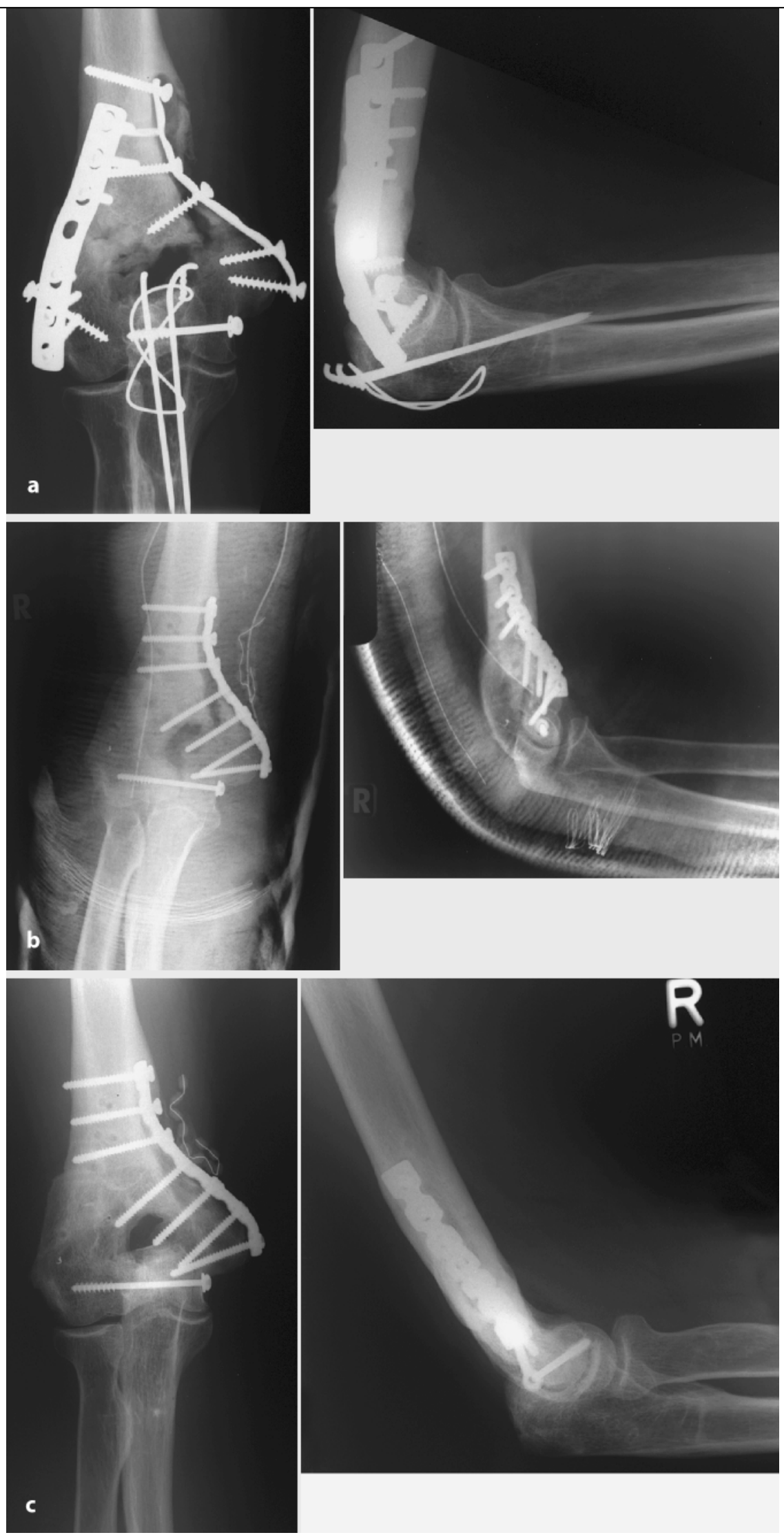

Abb. 2 \ 54-jähriger Patient, a Pseudarthrose des ulnaren Epikondylus nach C-Fraktur und auswärtig durchgeführter bikondylärer, instabiler Plattenosteosynthese, $\mathbf{b}$ postoperativer radiologischer Befund nach Restabilisierung und autologer Spongiosaplastik, $\mathbf{c}$ radiologisches Ausheilungsergebnis nach 6 Monaten 

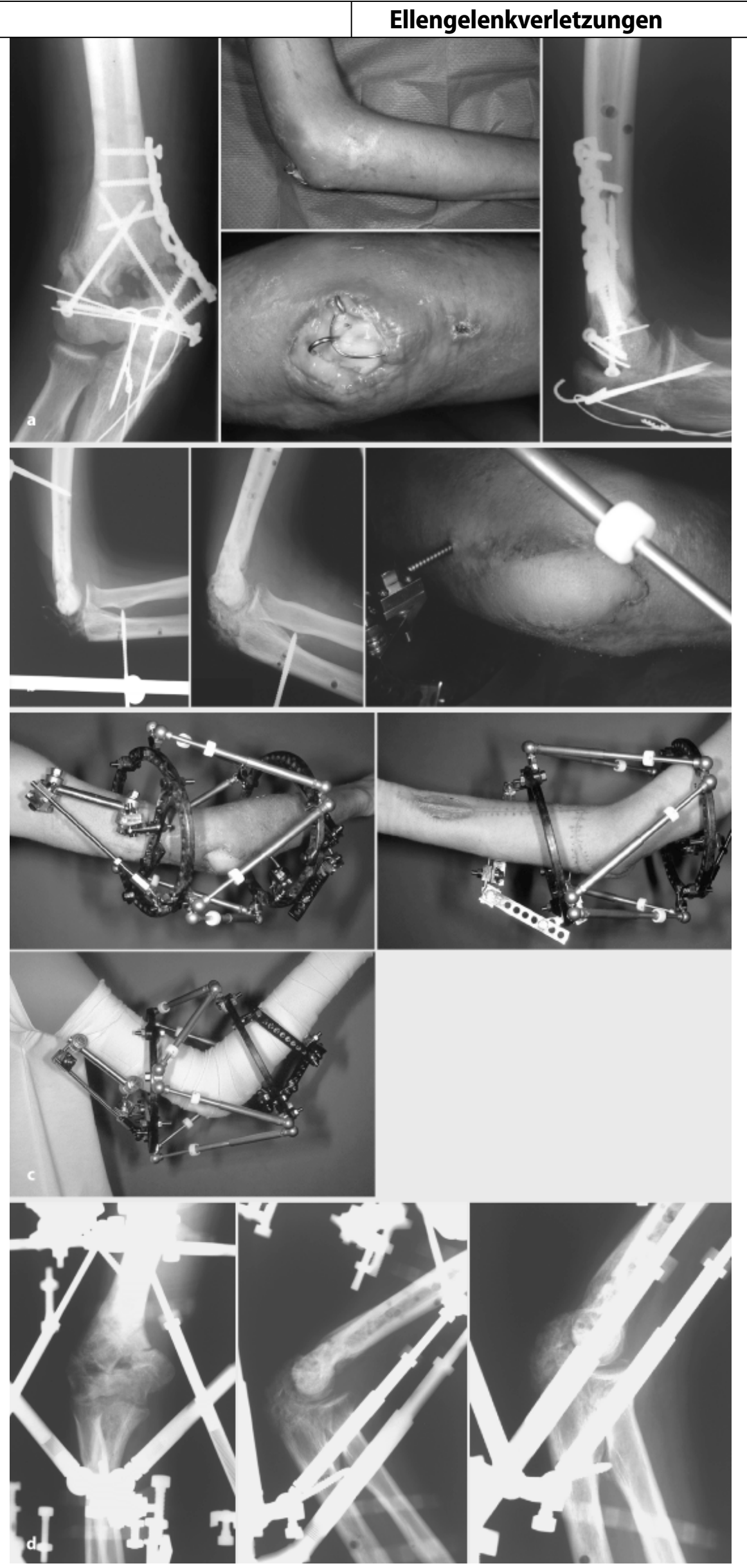

Die korrekturbedürftigen Fehlstellungen sind sehr selten und ebenfalls auf eine inadäquate operative Versorgung zurückzuführen [9, 23]. Pseudarthrosen gehen häufig mit einer erheblichen Gelenksteife einher, was die Behandlung erschwert, weil einerseits die postoperative Ruhigstellung (zur Behandlung der Pseudarthrose), anderseits eine frühfunktionelle Nachbehandlung (Behandlung der Gelenksteife) erforderlich sind. Aufgrund eigener Erfahrung sollte bei ausreichender interfragmentärer Stabilität eine begrenzte Bewegungsübung möglich sein.

Die Operationstechnik bei Pseudarthrosen ist überwiegend gleich. Der dorsale Zugang, der auch bei der frischen Frakturversorgung Anwendung findet, erlaubt die radiale und ulnare Exposition des distalen Humerus entlang der Trizepssehne. Ist eine Neurolyse indiziert, kann sie ebenfalls in Bauchlage erfolgen. Eine Olekranonosteotomie ist überwiegend bei intraartikularen Korrekturen notwendig, kann aber bei suprakondylärer Pseudarthrosenlage hilfreich sein.

Überwiegend findet sich die extraartikulare und unikondyläre Pseudarthrose. In diesen Fällen bedarf es ausschließlich einer radialen oder ulnaren Exposition in Rückenlage. Bei suprakondylärer Lage der Pseudarthrose ist eine bikondyläre Plattenosteosynthese im $90^{\circ}$-Winkel mit LCDC-Platten anzustreben. Winkelstabile Platten können hier zusätzliche Sicherheit gewährleisten. Zudem ist es notwendig, bei Defekten eine autologe Spongiosaplastik durchzuführen.

\section{Posttraumatische Ellengelenkempyeme}

Infektionen des Ellengelenks nach Trauma sind zwar vergleichsweise selten, bedeuten aber für den Erfolg der primären Thera-

Abb. $3 \varangle$ 20-jähriger Patient, a chronisches Ellengelenkempyem und Osteitis des Olekranons mit Hautweichteildefekt nach ehemaliger drittgradig offener supra- und diakondylärer Oberarmfraktur, primärer Fixateur-externe-Anlage, sekundärer Plattenosteosynthese; $\mathbf{b}$ radiologischer und klinischer Befund nach Sequestrektomie und sekundärer spongiöser Aufbauplastik des Olekranons und freiem Radialislappen zur Defektdeckung; c klinische, $\mathbf{d}$ radiologische Befunde der Mobilisationsbehandlung im Hexapoden über 3 Monate; 
pie und die Gelenkfunktion eine erhebliche Gefährdung und erfordern deshalb rasches und konsequentes Handeln. Sie können grundsätzlich bei offenen Frakturen und/oder Luxationen durch bakterielle Einsaat und als Komplikation nach operativen Maßnahmen eintreten.

Die verschiedenen Klassifikationen der Gelenkinfektion sind bekannt und sollen an dieser Stelle nicht weiter aufgeführt werden $[28,29,31]$.

Das therapeutische Vorgehen erfolgt In Übereinstimmung mit der Literatur stadienadaptiert [7, 27, 28, 29, 31].

Als therapeutische Empfehlung der Gelenkinfektion nach posttraumatischen Primäreingriffen ist die bilaterale $\mathrm{Ar}$ throtomie zu nennen. Inwieweit die einliegende Osteosynthese nicht entfernt werden muss, ist vom Einzelfall abhängig. Liegen infizierte Osteosynthesen regelrecht und stabil ein, können sie nach Überprüfung der Stabilität belassen werden. Nach Schmidt [27] und Schmidt et al. [28] gelingt in etwa 50\% der Fälle ein Erhalt des Osteosynthesematerials bei zusätzlicher Einlage eines lokalen Antibiotikumträgers. Finden sich jedoch eine Lockerung oder Fehllage der Osteosynthese, muss diese entfernt werden. Des Weiteren muss intraoperativ entschieden werden, ob eine zusätzliche Transfixation des $\mathrm{Ge}$ lenks (Fixateur externe) erforderlich ist.

Nach Schmidt et al. [29] kann zusammenfassend festgestellt werden:

- Je einfacher die Fraktur und je übersichtlicher und fehlerfreier die Osteosynthese sind, desto eher wird der Erhalt der internen Osteosynthese möglich sein.

- Je komplexer die Fraktur und die Versorgung und je fortgeschrittener die Infektion sind, desto eher fällt die Entscheidung zur Metallentfernung und zur anschließenden Transfixation im Fixateur externe.

Ziel jeglicher Behandlung an der oberen Extremität muss der Wiedergewinn der Funktion sein. Die Stabilität ist nur das 2. Ziel. Daher sollte eine Arthrodese des Ellengelenks nur bei hochgradiger Zerstörung, größeren Knochendefekten und gleichzeitig bestehender foudroyanter Infektion vorgenommen werden. In den häufigeren Fällen, in welchen die Infektion durch das oben
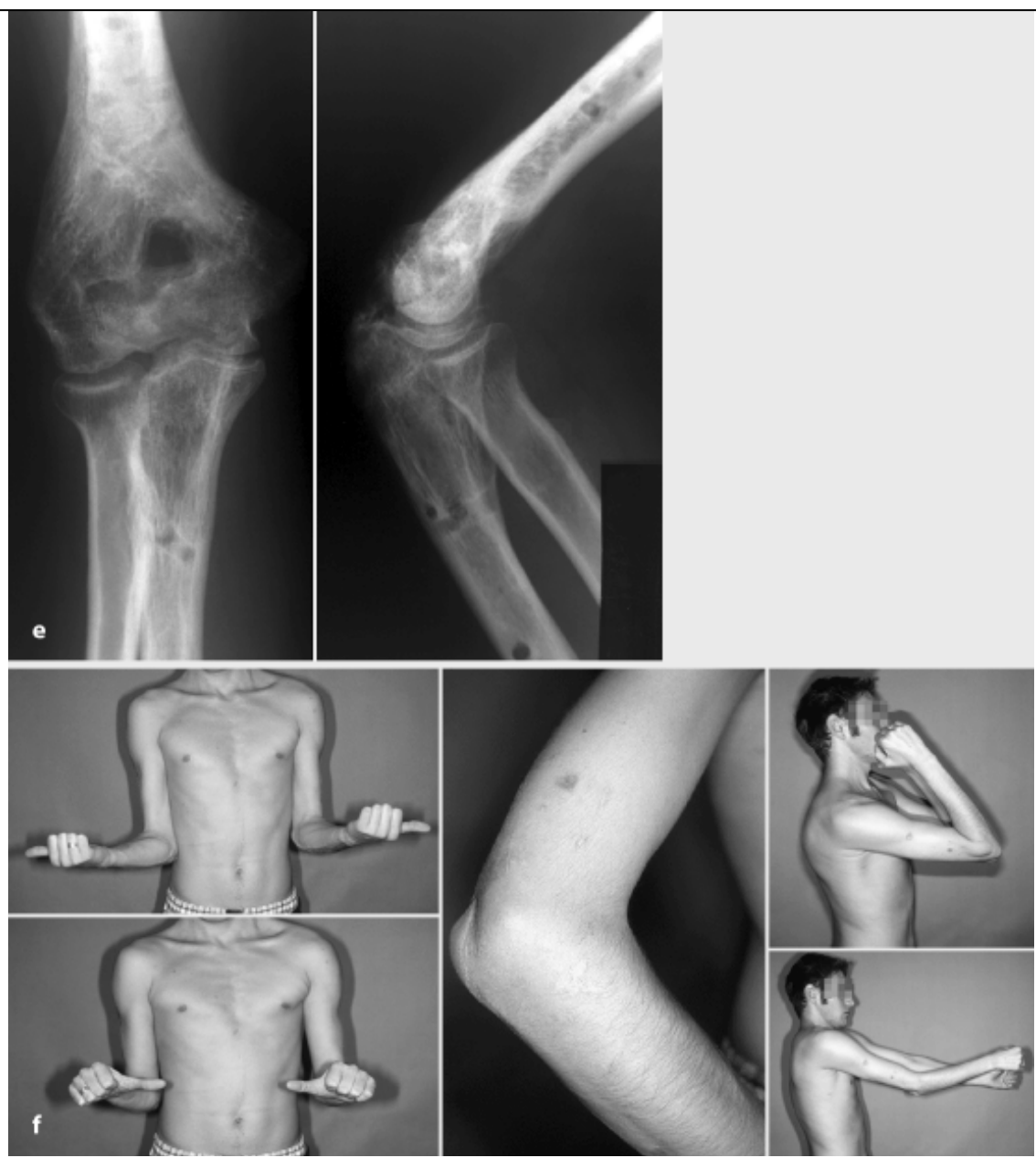

Abb. 3 \ 20-jähriger Patient, e radiologisches Ausheilungsergebnis, $\mathbf{f}$ funktionelles Ergebnis nach 7 Monaten

geschilderte Vorgehen beruhigt werden konnte, auch wenn schwerwiegende Knorpelschäden vorlagen, ist trotz weitgehender Gelenkzerstörung hier einer Gelenkresektion der Vorzug zu geben, weil dadurch zumindest eine Teilgelenkfunktion wiederhergestellt werden kann (• Abb. 3).

\section{Endoprothetik}

Diese Möglichkeit kann bei destruiertem Ellenbogengelenk bei korrekter Indikationsstellung einen erheblichen Zuwachs an Lebensqualität für den Patienten darstellen (- Abb. 4). Die bisherigen guten Ergebnisse bei rheumatoider Arthritis können nicht auf die Behandlung der posttraumatischen Arthrose oder Fehlstellung übertragen werden, da sich insbesondere das Patientenkollektiv deutlich unterscheidet. Bei der posttraumatischen Ellengelenkdestruktion handelt es sich häufig um Zustände nach sehr komplexen, Kno- chen und Weichteile in Mitleidenschaft ziehenden Frakturen oder Luxationsfrakturen. Bei den posttraumatischen Arthrosen findet sich ein höherer Prozentsatz an relativ jungen männlichen Patienten, welche höhere funktionelle Ansprüche an den operierten Ellenbogen stellen. Hieraus kann (mit der größeren Lebenserwartung des jüngeren Patienten) ein höherer Prozentsatz an Versagern (v. a. aseptischen Lockerungen) resultieren.

In der Literatur $[11,16]$ werden Komplikationsraten von 14-50\% aufgeführt. Nach Mansat u. Morrey [16] stellt der endoprothetische Gelenkersatz bei eingesteiften Ellenbogengelenken eine chirurgische Behandlungsoption der letzten Wahl dar mit relativ hoher Komplikationsrate und eingeschränkten funktionellen Ergebnissen. Sie konnten aufzeigen, dass bei einer Analyse von 14 Patienten eine Verbesserung des Bewegungsausmaßes von ausschließlich $60^{\circ} \mathrm{zu}$ erzielen war. 

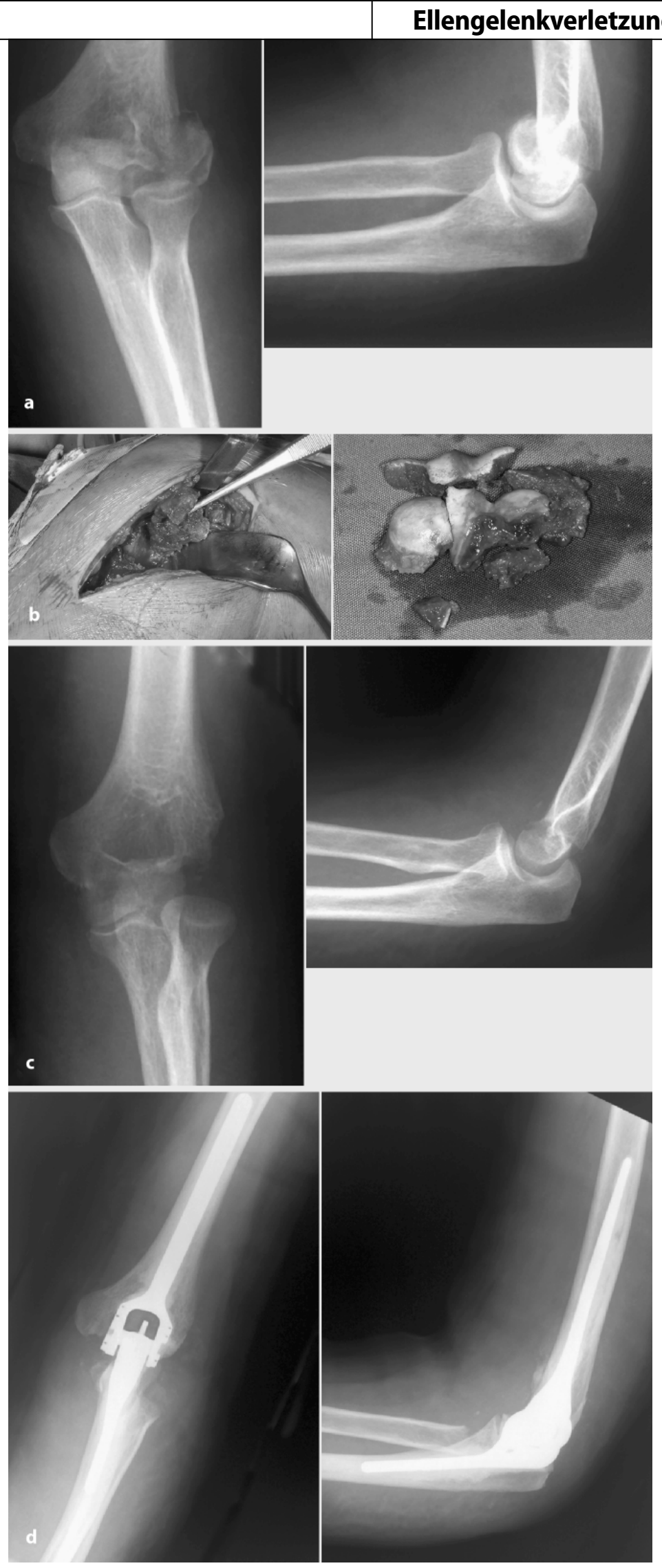

Abb. 4 \ 50-jährige Patientin, a Mehrfragmentfraktur des radialen Epikondylus; b intraoperativer Befund mit multiplen Fragmenten, die adäquate Osteosynthese verhindernd; c, d radiologischer Befund $\mathbf{c}$ nach Resektion, $\mathbf{d}$ nach sekundärer Implantation einer Endoprothese

Grundsätzlich werden 2 große Gruppen von Ellenbogenkunstgelenken unterschieden:

- verblockte („linked“) Prothesen und

- unverblockte („un-linked“) Prothesen.

Erstere sind Scharniergelenke, die nur noch ausnahmsweise bei malignen $\mathrm{Tu}$ moren als starre Scharniergelenke zur Anwendung kommen. Die überwiegenden Prothesenmodelle sind aktuell so genannte laxe Scharniergelenke („sloppy hings"), welche ein Spiel zwischen beiden Komponenten erlauben. Die Scharniergelenke benötigen zur stabilen Fixation im Knochen Markraumschäfte und Knochenzement.

Unverblockte Prothesen ersetzen die gelenkigen Oberflächen von Humerus und Ulna (nur in Ausnahmefällen auch diejenigen des Radius) in Form von zylindrischen Konfigurationen oder in Annäherung an die anatomische Gestalt des Ellenbogengelenks.

In Bezug auf die Indikationsstellung und auch die zu erwartenden Ergebnisse ergeben sich Unterschiede zwischen verblockten und unverblockten Prothesen. Bei Letzteren bedarf es eines intakten Kapsel-Band-Apparats sowie Gelenkepicondylen. Bei größeren Defekten oder fehlendem Bandapparat sinken die Chancen für eine unverblockte Prothese. Bei posttraumatischen Zuständen haben sich die so genannten "sloppy hings“ gegenüber ihnen als eindeutig überlegen gezeigt [12].

Loehr et al. [12] berichteten über 21 Fälle, bei denen primär nach Frakturen oder Luxationsfrakturen Prothesen implantiert wurden. Die Indikation bestand überwiegend bei sehr alten Patienten, bei welchen eine ausgeprägte Osteoporose die Erzielung einer stabilen Reposition und inneren Fixation als wenig aussichtsreich erschienen ließ. Bei einer Untersuchung nach 4 Jahren waren alle Patienten hinsichtlich der Schmerzen zufrieden. Objektiv konnten 15 Patienten als sehr gut und 5 als gut bewertet werden.

In Übereinstimmung mit der Literatur $[11,21]$ darf der endoprothetisch versorgte Ellenbogen keine größeren Gewichte tragen oder schwere Arbeiten ver- 
richten. Bei entsprechenden Patienten sollte kein endoprothetischer Gelenkersatz erfolgen.

\section{Korrespondierender Autor}

Dr. S. Fuchs

Abteilung für Unfall- und Wiederherstellungs-

chirurgie, BG-Unfallkrankenhaus

Bergedorfer Straße 10, 21033 Hamburg

S.Fuchs@buk-hamburg.de

Interessenkonflikt. Es besteht kein Interessenkonflikt. Der korrespondierende Autor versichert, dass keine Verbindungen mit einer Firma, deren Produkt in dem Artikel genannt ist, oder einer Firma, die ein Konkurrenzprodukt vertreibt, bestehen. Die Präsentation des Themas ist unabhängig und die Darstellung der Inhalte produktneutral.

\section{Literatur}

1. Beickert R, Bühren V (2003) Arthrolyse des Ellengelenkes. Trauma Berufskrankh 5: 41-54

2. Eijer H, Schafroth M, Marti RK (2001) Die Behandlung von nicht oder nicht korrekt verheilten Frakturen am Ellenbogen. Orthopädie 30: 627-634

3. Exner G, Wisotzky W, Johne B et al. (1982) Therapie, insbesondere die Arthrolyse und deren Ergebnisse am versteiften Ellenbogengelenk nach Frakturen. Aktuelle Traumatol 12: 255-262

4. Hastings H, Graham T (1994) The classification and treatment of heteropic ossification about the elbow and forearm. Hand Clin 10:417-437

5. Hertel R, Wolfram L (2001) Offene Ellenbogenarthrolyse bei erhaltener Gelenkflächengeometrie. Orthopädie 30: 619-625

6. Jäger M, Wirth CJ (1981) Die Arthrolyse und Arthroplastik des Ellenbogen- und Kniegelenkes. Huber, Bern Stuttgart Toronto

7. Jerosch J, Prymka M (1998) Arthroskopische Therapie der septischen Arthritis - Operative Technik und Ergebnisse. Unfallchirurg 101: 454-460

8. Josten C, Lill H (2002) Ellenbogenverletzungen. Steinkopff, Darmstadt

9. Kaminski A, Müller EJ, Muhr G (2003) Korrekturoperationen nach fehlverheilten supra- und diakondylären Oberarmfrakturen. Trauma Beruskrankh 5: 24-28

10. Lahoda LU, Klapperich T, Hahn MP et al. (1999) Ergebnisse nach posttraumatischen Ellenbogengelenkarthrolysen. Chirurg 70: 1302-1306

11. Lerch $K$, Tingart M, Trail I et al. (2003) Der endoprothetische Gelenkersatz des Ellenbogengelenks. Orthopädie 32: 730-735

12. Loehr JF, Gschwend N, Simmen BR et al. (2003) Endoprothetik des Ellenbogens. Orthopädie 32: 717 722

13. Loew M (2001) Die Ellenbogenkontraktur. Orthopädie 30: 587-592

14. Mader K, Pennig D, Gausepohl T et al. (2004) Arthrolyse des Ellenbogengelenkes. Unfallchirurg 107: 403-414

15. Mansat P, Morrey BF (1998) The column procedura: a limited lateral approach for extrinsic contracture of the elbow. J Bone Joint Surg Am 80-A: 16031615

16. Mansat P, Morrey BF (2001) Endoprothetischer Gelenkersatz bei eingesteiftem Ellenbogengelenk. Orthopädie 30: 645-648
17. Mansat P, Morrey BF, Hotchkiss RN (2000) Extrinsic contracture "the column procedure", lateral und medial capsular release. In: Morrey BF (ed) The elbow and its disorders. Saunders, Philadelphia

18. Mohan K (1972) Myosititis ossificans of the elbow. Int Surg 57: 475-480

19. Morrey BF (1990) Post-traumatic contracture of the elbow. J Bone Joint Surg Am 72-A: 618

20. Morrey BF (2000) Surgical treatment of extraarticular elbow contracture. Clin Orthop 370: 57-64

21. Morrey BF (2003) Differenzialindikation zwischen Arthroplastik und Endoprothetik des rheumatischen Ellenbogengelenkes. Orthopädie 32: 1028-1037

22. Morrey BF, An KN, Chao EYS (1993) Functional evaluation of the elbow. In: Morrey BF (ed) The elbow and its disorders. Saunders, Philadelphia

23. Mutschler W, Burri C, Rübenacker S (1990) Rekonstruktive Chirurgie fehlverheilter Ellenbogengelenkbrüche. Orthopädie 19: 324-331

24. Pennig D, Mader K, Gausepohl T (2005) Bewegungseinschränkung nach Verletzung des Ellenbogengelenkes: Planung und operative Strategie der Arthrolyse. Zentralbl Chir 130: 32-40

25. Regel G, Seekamp A, Blauth M et al. (1998) Ellenbogennahe Pseudarthrosen, Ursachen und Behandlung. Unfallchirurg 101: 193-200

26. Sojbjerg JO (1996) The stiff elbow. Acta Orthop Scand 67: 626-631

27. Schmidt HGK (2003) Infekt des Ellengelenkes, was ist zu tun? Trauma Berufskh [Suppl 1] 2: S86-S91

28. Schmidt HGK, Gerlach U, Wurm M et al. (2001) Diagnostik und Therapie von Schulter- und Ellengelenkempyemen. Trauma Berufskrankh [Suppl 3] 3: S404-S414

29. Schmidt HGK, Gerlach UJ, Hadler D et al. (2003) Therapie des posttraumatischen Ellengelenkempyems. Trauma Berufskrankh 5: 55-64

30. Strobel MJ, Eckardt OA, Eichhorn HJ (2001) Die arthroskopische Therapie bei Bewegungseinschränkungen des Ellenbogengelenkes. Orthopäde 30: 610-618

31. Stutz A, Gächter A (2001) Diagnostik und stadiengerechte Therapie von Gelenkinfekten. Unfallchirurg 104: 682-686 